\title{
Erratum to: Widespread endocrine activity in river sediments in Hesse, Germany, assessed by a combination of in vitro and in vivo bioassays
}

\author{
Simone Galluba • Jörg Oehlmann
}

Published online: 11 January 2012

(C) Springer-Verlag 2011

Erratum to: J Soils Sediments

DOI 10.1007/s11368-011-0454-0
Table 2: The values highlighted in light grey were missing in this table.

Table 2 Contamination levels at sampling sites expressed as sums of quality limit value exceedances

\begin{tabular}{|c|c|c|c|c|c|c|}
\hline $\begin{array}{l}\text { Sampling } \\
\text { site }\end{array}$ & Metals & PCB & PAH & OTC & Total & \multirow{24}{*}{$\begin{array}{l}\text { No or very low } \\
\text { contamination } \\
\text { Excessive } \\
\text { contamination }\end{array}$} \\
\hline 9 & 2.32 & 0 & 6.05 & 0 & 8.37 & \\
\hline 10 & 0 & 0 & 4.15 & 0 & 4.15 & \\
\hline 15 & 0 & 0 & 3.95 & 0 & 3.95 & \\
\hline 16 & 0 & 0 & 3.05 & 0 & 3.05 & \\
\hline 18 & 1.06 & 0 & 40.8 & 1.30 & 43.2 & \\
\hline 20 & 10.3 & 0 & 12.8 & 0 & 23.1 & \\
\hline 21 & 0 & 0 & 3.10 & 0 & 3.10 & \\
\hline 22 & 0 & 0 & 0 & 0 & 0 & \\
\hline 23 & 3.36 & 2.45 & 19.8 & 1.35 & 26.9 & \\
\hline 24 & 7.07 & 2.75 & 7.65 & 0 & 17.5 & \\
\hline 25 & 2.90 & 1.15 & 19.4 & 0 & 23.5 & \\
\hline 26 & 2.62 & 0 & 10.5 & 0 & 13.1 & \\
\hline 28 & 3.00 & 1.05 & 33.4 & 0 & 37.4 & \\
\hline 31 & 15.3 & 2.40 & 5.40 & 0 & 23.1 & \\
\hline 32 & 1.09 & 0 & 0 & 0 & 1.09 & \\
\hline 33 & 1.21 & 0 & 4.40 & 0 & 5.61 & \\
\hline 34 & 1.35 & 0 & 13.6 & 0 & 15.0 & \\
\hline 36 & 3.57 & 0 & 3.10 & 0 & 6.67 & \\
\hline 39 & 14.0 & 17.7 & 6.75 & 0 & 38.4 & \\
\hline 41 & 5.95 & 0 & 0 & 1.11 & 7.06 & \\
\hline 43 & 2.26 & 0 & 5.60 & 0 & 7.86 & \\
\hline 44 & 3.95 & 0 & 6.95 & 12.5 & 23.4 & \\
\hline 47 & 2.59 & 0 & 3.40 & 0 & 5.99 & \\
\hline 48 & 2.20 & 0 & 5.10 & 0 & 7.30 & \\
\hline
\end{tabular}

Contamination levels (represented by shades of grey) are in steps of $20 \%$, with $100 \%$ representing the highest contamination of a substance class (for details see Table 1). For sampling sites not listed here, no chemical analytical data were available.

The online version of the original article can be found at http://dx. doi.org/10.1007/s11368-011-0454-0.

S. Galluba $(\bowtie) \cdot$ J. Oehlmann

Department of Aquatic Ecotoxicology,

Goethe University Frankfurt am Main,

Max-von-Laue-Str. 13,

60438 Frankfurt am Main, Germany

e-mail: galluba@bio.uni-frankfurt.de 